\title{
Electroencephalogram Background Activity Characterization with Detrended Moving Average in Alzheimer's Disease Patients
}

\author{
Daniel Abásolo ${ }^{1}$, Member, IEEE, Roberto Hornero ${ }^{1}$, Member, IEEE, Carlos Gómez ${ }^{1}$, Student \\ Member, IEEE, Javier Escudero ${ }^{1}$, Student Member, IEEE, and Pedro Espino ${ }^{2}$ \\ ${ }^{1}$ Biomedical Engineering Group, E.T.S.I. de Telecomunicación, University of Valladolid, Camino del \\ Cementerio s/n, 47011, Valladolid, Spain (corresponding author's e-mail: daniel.abasolo@tel.uva.es; phone: \\ +34983423981; fax: +34983423667). \\ ${ }^{2}$ Hospital Clínico San Carlos, c/Profesor Martín Lagos s/n, 28040, Madrid, Spain.
}

\begin{abstract}
The aim of this study was to analyse the electroencephalogram (EEG) background activity in Alzheimer's disease (AD) with the Detrended Moving Average (DMA) method, a new approach to quantify correlation properties in non-stationary signals with underlying trends. EEGs were recorded from the 19 scalp loci of the international 10-20 system in $11 \mathrm{AD}$ patients and 11 age-matched controls. Our results showed two scaling regions in all subjects' channels, with a clear bend when their corresponding slopes $\left(\alpha_{1}\right.$ and $\left.\alpha_{2}\right)$ were distinctly different. With the exception of electrode $\mathrm{T} 4$, the $\alpha_{1}$ values were lower in control subjects than in AD patients, with significant differences at T5, P3, P4 and $O 1$ ( $p<0.01$, Student's $t$-test). On the other hand, $\alpha_{2}$ values were higher in control subjects than in AD patients, with significant differences only at F4. Furthermore, we evaluated the ability of $\alpha_{2}$ to discriminate AD patients from control subjects at these electrodes using ROC plots. We obtained a maximum accuracy of $81.82 \%$ at $\mathrm{O} 1$ with $\alpha_{1}$ and at $F 4$ with $\alpha_{2}$. These findings suggest that the scaling behaviour of the EEG is sensitive to $\mathrm{AD}$ and that the DMA method could help to increase our insight into brain dysfunction in AD.
\end{abstract}

\section{INTRODUCTION}

A LZHEIMER'S disease (AD) is a primary degenerative dementia of unknown aetiology that gradually destroys brain cells and represents the most prevalent form of dementia in western countries [1]. AD is characterized by progressive impairments in cognition and memory whose course lasts several years prior to the death of the patient [2]. Structural changes in $\mathrm{AD}$ are related to the accumulation of amyloid plaques between nerve cells in the brain and with the appearance of neurofibrillary tangles inside nerve cells, particularly in the hippocampus and the cerebral cortex [3].

The clinical diagnosis of AD is made primarily on the basis of medical history studies, psychiatric evaluation and different memory, reasoning and mental status tests. However,

This work was supported by the grant project GR-150 (Network of excellence) from Junta de Castilla y León and by the grant project TEC200802241 from Ministerio de Ciencia e Innovación. physicians only make a diagnosis of $\mathrm{AD}$ with an accuracy of about $90 \%$ and a definite diagnosis is only possible by necropsy [4]. Hence, new approaches are necessary to improve $\mathrm{AD}$ diagnosis.

The electroencephalogram (EEG) has been used as a tool for investigating dementias for several decades. This is mainly due to the fact that $\mathrm{AD}$ is a cortical dementia in which EEG abnormalities are more frequently shown. AD patients' EEGs generally show a shift of the power spectrum to lower frequencies and a decrease of coherence among cortical areas [2]. However, in the early stages of the disease the EEG may exhibit normal frequencies and may be similar to that of elderly control subjects [5]. From another point of view, several studies have examined the non-linear dynamics of the EEG in $\mathrm{AD}$ (a detailed review can be found in [2]). In general, the EEG is less complex and more regular in $\mathrm{AD}$ patients than in controls [2], [6], [7]. Moreover, AD patients' EEGs show reduced functional connections when compared to elderly controls [8]. EEG abnormalities in AD are thought to be associated with functional disconnections among cortical areas resulting from death of cortical neurons, axonal pathology, cholinergic deficits, etc. [2].

The complex nature of the electrical brain activity results in fluctuations in the EEG [9]. To understand EEG activity in a better way, it is necessary to characterize its fluctuations over different time scales. Recent studies show that EEG oscillations in the human brain show long-range temporal correlations [9]-[11] and that the scaling behaviour of the EEG is sensitive to $\mathrm{AD} \mathrm{[12].}$

In this preliminary study, we examined the scaling behaviour of the EEG in AD with the Detrended Moving Average (DMA) method, a new approach to quantify correlation properties in non-stationary signals with underlying trends [13]. We wanted to test the hypothesis that long-range temporal correlations in AD patients' EEGs would be different from those of age-matched controls. 


\section{MATERIAL AND METHODS}

\section{A. Subjects and EEG recording}

Twenty-two subjects participated in this study. Eleven patients ( 5 men and 6 women; age $=72.5 \pm 8.3$ years, mean \pm standard deviation, SD) fulfilling the criteria of probable AD were recruited from the Alzheimer's Patients' Relatives Association of Valladolid (AFAVA) and referred to the University Hospital of Valladolid (Spain), where the EEG was recorded. All of them had undergone a thorough clinical evaluation that included clinical history, physical and neurological examinations, brain scans and a Mini-Mental State Examination (MMSE), generally accepted as a quick and simple way to evaluate cognitive function [14]. The mean MMSE score for the patients was $13.1 \pm 5.9$. The control group consisted of 11 age-matched control subjects without past or present neurological disorders ( 7 men and 4 women; mean age $=72.8 \pm 6.1$ years). The local ethics committee approved the study and all control subjects and all caregivers of the patients gave their informed consent for participation in the current study.

EEGs were recorded from the 19 scalp loci of the international 10-20 system (electrodes F3, F4, F7, F8, Fp1, Fp2, T3, T4, T5, T6, C3, C4, P3, P4, O1, O2, Fz, Cz and Pz) using a Profile Study Room 2.3.411 EEG equipment (Oxford Instruments). More than five minutes of data were recorded from each subject. The sample frequency was $256 \mathrm{~Hz}$, with a 12-bit A-to-D precision. Recordings were made under the eyes-closed condition in order to obtain as many artefact-free EEG data as possible. All EEGs were visually inspected by a specialist physician to check for eye movement and other artefacts. Afterwards, EEGs were organized in 5 second artefact-free epochs (1280 points) that were copied as ASCII files for off-line analysis on a personal computer. Furthermore, all recordings were digitally filtered with a band-pass filter with cut-off frequencies at $0.5 \mathrm{~Hz}$ and at 40 $\mathrm{Hz}$ in order to remove EMG activity prior to the computation of DMA.

\section{B. Detrended Moving Average}

Detrended Fluctuation Analysis (DFA) provides an estimation of scaling information and long-range correlations in time series, and is known for its robustness against nonstationarities [9], [15]. However, as the removal of trends in $D F A$ is based on discontinuous polynomial fitting, oscillations in the fluctuation function and significant errors in crossover locations can be introduced. The Detrended Moving Average $(D M A)$ method was introduced to estimate correlation properties of non-stationary signals without any assumption of the type of trends, the probability distribution or other characteristics of the underlying process [16].

Let the EEG time series be denoted by $\{x(i)\}$, where $i$ is the discrete time, ranging from $i=1$ to $N(N=1280)$. To perform the DMA method, first we need to integrate the EEG time series to obtain:

$$
y(i)=\sum_{j=1}^{i}[\{x(j)\}-\bar{x}]
$$

where $\bar{x}$ is the mean of the whole temporal series. Then, trends are detected in the data with a moving average.

There are two important categories of moving average: simple moving average and weighted moving average. In this study we consider the simple moving average, where equal weight is assigned to each data point in a window of size $n$. The position to which the average of all weighted data points is assigned determines the relative contribution of the "past" and "future" points [16]. We consider the backward moving average, which for a window of size $n$ is defined as

$$
\tilde{y}_{n}(i)=\frac{1}{n} \sum_{k=0}^{n-1} y(i-k),
$$

where $y(i)$ is the aforementioned integrated signal. The average of the signal data points within the window refers to the last sample covered by the window. Thus, the operator in (2) is causal.

Once the moving average is obtained, we detrend the signal by subtracting it from the integrated profile:

$$
C_{n}(i)=y(i)-\tilde{y_{n}}(i)
$$

We then calculate the fluctuation for a window of size $n$ as

$$
F(n)=\sqrt{\frac{1}{N-n+1} \sum_{i=1}^{N}\left[C_{n}(i)\right]^{2}} .
$$

A power law relation between the fluctuation function $F(n)$ and the scale $n \quad\left(F(n) \propto n^{\alpha}\right)$ indicates a self-similar behaviour.

\section{Statistical analysis}

Student's $t$-test was used to evaluate the statistical differences between the scaling exponents from AD patients and control subjects. Differences were considered statistically significant if the $p$ value was lower than 0.01 .

The ability to discriminate AD patients from control subjects at the electrodes where $p<0.01$ was evaluated using Receiver Operating Characteristic (ROC) curves [17].

\section{RESULTS}

We performed the DMA method for channels F3, F4, F7, F8, Fp1, Fp2, T3, T4, T5, T6, C3, C4, P3, P4, O1, and O2. We studied the fluctuations using window sizes between 3 and 
128 samples (from $0.01 \mathrm{~s}$ to $0.5 \mathrm{~s}$ ). Furthermore, we plotted the natural logarithm of $F(n)$ as a function of the natural logarithm of $n$. If the plot displays a linear scaling region with a certain scaling exponent, then there is a power-law behaviour in the time series.

We found two scaling regions in the EEG with a clear bend when the two slopes in the two regions are distinctly different. These scaling properties were found in all channels for all subjects. Fig. 1 and Fig. 2 show $F(n)$ vs. $n$ in a log-log plot for one EEG epoch from a control subject at electrode T5 and for one EEG epoch from an AD patient at electrode T5, respectively. In both cases, two different slopes can be seen and a bend in the transition between them can be observed. We have denoted the scaling exponent of the first region as $\alpha_{1}$ and the exponent on the second one as $\alpha_{2}$.

To quantify the scaling exponents, we performed a linear fit in region I for $1<\ln n<2.3$ and in region II for $3<\ln n<$ 4.5. The limits were chosen after a visual inspection of the results. The $\alpha_{1}$ and $\alpha_{2}$ values for the AD patients and control

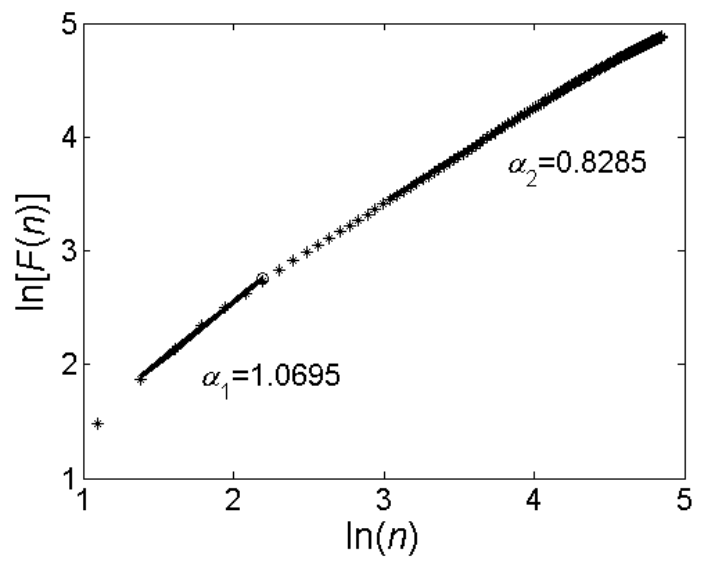

Fig. 1. $F(n)$ vs. $n$ in a $\log -\log$ plot for one EEG epoch from electrode T5 of a control subject. The scaling exponents $\alpha_{1}$ and $\alpha_{2}$ are depicted with a solid line and their numerical values included.

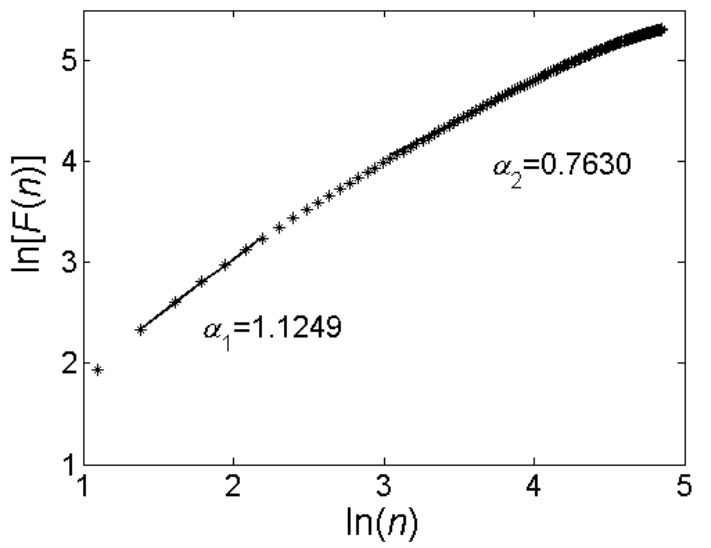

Fig. 2. $F(n)$ vs. $n$ in a log-log plot for one EEG epoch from electrode T5 of an $\mathrm{AD}$ patient. The scaling exponents $\alpha_{1}$ and $\alpha_{2}$ are depicted with a solid line and their numerical values included. subjects and the $p$ values of the Student's $t$-tests performed to examine the differences between both groups are summarized in Tables I and II, respectively. With the exception of electrode T4, the $\alpha_{1}$ values were lower in control subjects than in AD patients, with significant differences at T5, P3, P4 and O1 $(p<0.01)$. On the other hand, $\alpha_{2}$ values were higher in control subjects than in $\mathrm{AD}$ patients, with significant differences only at F4.

Finally, we evaluated the ability of the scaling exponents to discriminate $\mathrm{AD}$ patients from control subjects at the electrodes where significant differences were found using ROC plots. Results are shown in Tables III and IV, where it can be seen that an accuracy of $81.82 \%$ was obtained at $\mathrm{O} 1$ with $\alpha_{1}$ and at F4 with $\alpha_{2}$.

TABLE I

AVerage Values of the SLOPE IN THE First Scaling Region. Significant DIFFERENCES ARE MARKED WITH AN ASTERISK.

\begin{tabular}{cccc}
\hline \hline Electrode & $\begin{array}{c}\text { Control subjects } \\
(\text { mean } \pm \text { SD) }\end{array}$ & $\begin{array}{c}\text { AD patients } \\
(\text { mean } \pm \text { SD) }\end{array}$ & $p$-value \\
\hline F3 & $1.1230 \pm 0.0422$ & $1.1433 \pm 0.0241$ & 0.1808 \\
F4 & $1.1268 \pm 0.0443$ & $1.1316 \pm 0.0233$ & 0.7536 \\
F7 & $1.1155 \pm 0.0414$ & $1.1286 \pm 0.0314$ & 0.4109 \\
F8 & $1.1185 \pm 0.0301$ & $1.1306 \pm 0.0241$ & 0.3112 \\
Fp1 & $1.1294 \pm 0.0323$ & $1.1525 \pm 0.0285$ & 0.0903 \\
Fp2 & $1.1306 \pm 0.0417$ & $1.1545 \pm 0.0219$ & 0.1072 \\
T3 & $1.0606 \pm 0.0697$ & $1.0730 \pm 0.0800$ & 0.7007 \\
T4 & $1.0748 \pm 0.0656$ & $1.0521 \pm 0.1390$ & 0.6300 \\
T5* & $1.0723 \pm 0.0533$ & $1.1304 \pm 0.0382$ & 0.0082 \\
T6 & $1.0818 \pm 0.0502$ & $1.1286 \pm 0.0403$ & 0.0257 \\
C3 & $1.0942 \pm 0.0533$ & $1.1191 \pm 0.0420$ & 0.2390 \\
C4 & $1.0893 \pm 0.0519$ & $1.1082 \pm 0.0542$ & 0.4144 \\
P3* & $1.0862 \pm 0.0462$ & $1.1412 \pm 0.0323$ & 0.0042 \\
P4* & $1.0850 \pm 0.0401$ & $1.1345 \pm 0.0327$ & 0.0048 \\
O1* & $1.0596 \pm 0.0481$ & $1.1270 \pm 0.0447$ & 0.0028 \\
O2 & $1.0712 \pm 0.0508$ & $1.1290 \pm 0.0447$ & 0.0102 \\
\hline \hline
\end{tabular}

TABLE II

Average VAlues of THE SLOPE IN THE SECOND SCALING REGION. SIGNIFICANT DIFFERENCES ARE MARKED WITH AN ASTERISK.

\begin{tabular}{cccc}
\hline \hline Electrode & $\begin{array}{c}\text { Control subjects } \\
(\text { mean } \pm \text { SD })\end{array}$ & $\begin{array}{c}\text { AD patients } \\
(\text { mean } \pm \text { SD) }\end{array}$ & $p$-value \\
\hline F3 & $0.8876 \pm 0.0479$ & $0.8079 \pm 0.1125$ & 0.0429 \\
F4* & $0.8974 \pm 0.0478$ & $0.7955 \pm 0.1032$ & 0.0075 \\
F7 & $0.8741 \pm 0.0433$ & $0.8187 \pm 0.0943$ & 0.0923 \\
F8 & $0.8855 \pm 0.0384$ & $0.8263 \pm 0.1002$ & 0.0823 \\
Fp1 & $0.8947 \pm 0.0175$ & $0.8650 \pm 0.0990$ & 0.3386 \\
Fp2 & $0.8983 \pm 0.0257$ & $0.8621 \pm 0.0750$ & 0.1458 \\
T3 & $0.8593 \pm 0.0567$ & $0.7696 \pm 0.0933$ & 0.0131 \\
T4 & $0.8661 \pm 0.0706$ & $0.7715 \pm 0.1010$ & 0.0192 \\
T5 & $0.8007 \pm 0.0593$ & $0.7359 \pm 0.1014$ & 0.0823 \\
T6 & $0.8312 \pm 0.0690$ & $0.7508 \pm 0.1088$ & 0.0517 \\
C3 & $0.8551 \pm 0.0683$ & $0.7993 \pm 0.1092$ & 0.1662 \\
C4 & $0.8588 \pm 0.0459$ & $0.7774 \pm 0.1328$ & 0.0691 \\
P3 & $0.7992 \pm 0.0665$ & $0.7515 \pm 0.1094$ & 0.2305 \\
P4 & $0.7937 \pm 0.1126$ & $0.7437 \pm 0.1203$ & 0.3272 \\
O1 & $0.7798 \pm 0.0899$ & $0.7374 \pm 0.1144$ & 0.3451 \\
O2 & $0.8071 \pm 0.1084$ & $0.7516 \pm 0.1006$ & 0.2281 \\
\hline \hline
\end{tabular}


TABLE III

AVERAGE SENSITIVITY, SPECIFICITY AND ACCURACY VALUES OBTAINED WITH ROC CURVES FOR THE FIRST SCALING REGION. THE OPTIMUM THRESHOLD THAT PROVIDES THESE VALUES AND THE AREA UNDER THE ROC CURVE ARE ALSO INCLUDED.

\begin{tabular}{cccccc}
\hline \hline E & Threshold & $\begin{array}{c}\text { Sensitivity } \\
(\%)\end{array}$ & $\begin{array}{c}\text { Specificity } \\
(\%)\end{array}$ & $\begin{array}{c}\text { Accuracy } \\
(\%)\end{array}$ & AUC \\
\hline T5 & 1.0971 & 81.82 & 72.73 & 77.27 & 0.8099 \\
P3 & 1.0998 & 81.82 & 72.73 & 77.27 & 0.8678 \\
P4 & 1.1100 & 72.73 & 81.82 & 77.27 & 0.8595 \\
O1 & 1.0969 & 81.82 & 81.82 & 81.82 & 0.8595 \\
\hline \hline
\end{tabular}

E: ELECTRODE.

TABLE IV

AVERAGE SENSITIVITY, SPECIFICITY AND ACCURACY VALUES OBTAINED WITH ROC CURVES FOR THE SECOND SCALING REGION. THE OPTIMUM THRESHOLD THAT PROVIDES THESE VALUES AND THE AREA UNDER THE ROC CURVE ARE Also INCLUDED.

\begin{tabular}{cccccc}
\hline \hline E & Threshold & $\begin{array}{c}\text { Sensitivity } \\
(\%)\end{array}$ & $\begin{array}{c}\text { Specificity } \\
(\%)\end{array}$ & $\begin{array}{c}\text { Accuracy } \\
(\%)\end{array}$ & AUC \\
\hline F4 & 0.8770 & 90.91 & 72.73 & 81.82 & 0.8430 \\
\hline \hline
\end{tabular}

E: ELECTRODE.

\section{DISCUSSION AND CONCLUSIONS}

We analysed the EEG background activity of $11 \mathrm{AD}$ patients and 11 control subjects with the DMA method with backward moving average, a new approach to quantify correlation properties in non-stationary signals with underlying trends [13]. We found two different scaling regions in the EEG that depend on the window size $n$. The first region corresponds to small time scales (less than $0.04 \mathrm{~s}$ ) and could be characterised by a scaling exponent $\alpha_{1}$. The second one corresponds to time scales from $0.08 \mathrm{~s}$ to $0.43 \mathrm{~s}$ and could be described with an exponent $\alpha_{2}$.

We have found that $\alpha_{1}$ was significantly lower in the control subjects' EEG at electrodes T5, P3, P4 and O1 and that $\alpha_{2}$ was significantly higher in that group at F4. Furthermore, the ability of both scaling exponents to discriminate $\mathrm{AD}$ patients from control subjects at these electrodes was evaluated using ROC plots. We obtained accuracies ranging from $77.27 \%$ to $81.82 \%$. These findings suggest that the scaling behaviour of the EEG is sensitive to AD. From the view of many body systems, long-range temporal correlations of EEG should originate from the strong interactions of the neural cells. It is logical to assume that AD would weaken or even block the interactions.

We have previously analysed the same database with $D F A$ and we only found significant differences between $A D$ patients and control subjects at T5, T6 and $\mathrm{O} 1$ in the second scaling region used in this study [12]. It has been suggested that DMA is an improvement over DFA [18], as the former does not require dividing the series into non-overlapping windows. Instead, the DMA method detrends the series by subtracting a continuous function, the moving average. Additionally, DMA is more accurate since the moving average is a better low-pass filter when compared to the polynomial filter used for $D F A$ [18]. Thus, EEG analysis with the $D M A$ method might be more appropriate than with $D F A$. However, it should be considered that, while the main significant differences between both groups were found at the first scaling region with $D M A, D F A$ showed significant differences only at the second scaling region. It could be argued that, although the $D M A$ method might be more appropriate than $D F A$, both methods could provide complementary information. Hence, we should investigate whether the combined use of DFA and DMA could improve the results obtained independently with each technique.

Some limitations of this preliminary study must be mentioned. Firstly, the sample size was small. To prove the usefulness of these techniques as an $\mathrm{AD}$ diagnostic tool, this approach should be extended on a much larger patient population. Moreover, the backward moving average is affected by a rather slow reaction to changes in the signal, due to a delay of half the window size compared to the signal [16]. Thus, other moving average schemes should be evaluated to check their possible usefulness in the analysis of EEG background activity in AD. Furthermore, as AD diagnosis is only definite after necropsy, the sample may not fully represent this disease. Finally, the scaling properties of the EEG should be studied in depression or other dementias to verify if the reported changes are specific to AD.

In summary, our results indicate that the $D M A$ method could be useful in AD diagnosis. In future studies we should investigate whether the combination of information obtained with $D M A$ at several electrodes, or even the combination of results obtained with different techniques, could provide a better classification between $\mathrm{AD}$ patients and control patients.

\section{REFERENCES}

[1] T. D. Bird, "Alzheimer's disease and other primary dementias," in Harrison's Principles of Internal Medicine, E. Braunwald A. S. Fauci, D. L. Kasper, S. L. Hauser, D. L. Longo, and J. L. Jameson, Eds. New York: The McGraw-Hill Companies Inc, 2001, pp. 2391-2399.

[2] J. Jeong, "EEG dynamics in patients with Alzheimer's disease," Clin. Neurophysiol., vol. 115, pp. 1490-1505, Jul. 2004.

[3] D. J. Selkoe, "Cell biology of the amyloid beta-protein precursor and the mechanism of Alzheimer's disease," Annu. Rev. Cell. Biol., vol. 10, pp. 373-403, 1994.

[4] M. Rossor, "Alzheimer's disease," in Brain's Diseases of the Nervous System, M. Donaghy, Ed. Oxford: Oxford University Press, 2001, pp $750-754$.

[5] O. N. Markand, "Organic brain syndromes and dementias," in Current Practice of Clinical Electroencephalography, D. D. Daly and T. A. Pedley, Eds. New York, NY: Raven Press Ltd., 1990, pp. 401-423.

[6] D. Abásolo, R. Hornero, P. Espino, J. Poza, C. I. Sánchez, and R. de la Rosa, "Analysis of regularity in the EEG background activity of Alzheimer's disease patients with Approximate Entropy," Clin. Neurophysiol., vol. 116, pp. 1826-1834, Aug. 2005.

[7] D. Abásolo, R. Hornero, P. Espino, D. Álvarez, and J. Poza, "Entropy analysis of the EEG background activity in Alzheimer's disease patients," Physiol. Meas., vol. 27, pp. 241-253, Mar. 2006.

[8] J. Jeong, J. C. Gore, and B. S. Peterson, "Mutual information analysis of the EEG in patients with Alzheimer's disease," Clin. Neurophysiol., vol. 112, pp. 827-835, May 2001. 
[9] R. C. Hwa and T. C. Ferree, "Scaling properties of fluctuations in the human electroencephalogram," Phys. Rev. E, vol. 66, p. 021901, Aug. 2002.

[10] V. V. Nikulin and T. Brismar, "Long-range temporal correlations in alpha and beta oscillations: effect of arousal level and test-retest reliability," Clin. Neurophysiol., vol. 115, pp. 1896-1908, Aug. 2004.

[11] V. V. Nikulin and T. Brismar, "Long-range temporal correlations in electroencephalographic oscillations: relation to topography, frequency band, age and gender," Neuroscience, vol. 130, pp. 549-558, 2005.

[12] D. Abásolo, R. Hornero, J. Escudero, and P. Espino, "A study on the possible usefulness of detrended fluctuation analysis of the electroencephalogram background activity in Alzheimer's disease," IEEE Trans. Biomed. Eng., vol. 55, pp. 2171-2179, Sep. 2008.

[13] E. Alessio, A. Carbone, G. Castelli, and V. Frappietro, "Second-order moving average and scaling of stochastic time series," Eur. Phys. J. B, vol. 27, pp. 197-200, 2002.
[14] M. F. Folstein, S. E. Folstein, and P. R. McHugh, "Mini-mental state. A practical method for grading the cognitive state of patients for the clinician," J. Psychiatr. Res., vol. 12, pp. 189-198, Nov. 1975.

[15] C.-K. Peng, S. V. Buldyrev, S. Havlin, M. Simons, H. E. Stanley, and A. L. Goldberger, "Mosaic organization of DNA nucleotides," Phys. Rev. E, vol. 49, pp. 1685-1689, Feb. 1994.

[16] L. Xu, P. Ch. Ivanov, K. Hu, Z. Chen, A. Carbone, and H. E. Stanley, "Quantifying signals with power-law correlations: A comparative study of detrended fluctuation analysis and detrended moving average techniques," Phys. Rev. E, vol. 71, p. 051101, May 2005.

[17] M. H. Zweig and G. Campbell, "Receiver-Operating Characteristic (ROC) plots: a fundamental evaluation tool in clinical medicine," Clin. Chem., vol. 39, pp. 561-577, Apr. 1993.

[18] A. Serletis and A. A. Rosenberg, "The Hurst exponent in energy futures prices," Physica A, vol. 380, pp. 325-332, 2007. 